Article

\title{
Outstanding Tensile Properties and Their Origins in Twinning-Induced Plasticity (TWIP) Steels with Gradient Substructures
}

\author{
Huihui Zhi ${ }^{1,2}$, Cheng Zhang ${ }^{1,2}$, Zihui Guo ${ }^{1,2}$, Stoichko Antonov ${ }^{3}$ and Yanjing Su ${ }^{1,2, *}$ \\ 1 Beijing Advanced Innovation Center for Materials Genome Engineering, University of Science and \\ Technology Beijing, Beijing 100083, China; huihui.zhi@hotmail.com (H.Z.); zhangchengustb@163.com (C.Z.); \\ guo_zihui@126.com (Z.G.) \\ 2 Corrosion and Protection Center, University of Science and Technology Beijing, Beijing 100083, China \\ 3 Department of Microstructure Physics and Alloy Design, Max-Planck-Institut für Eisenforschung, \\ 40237 Düsseldorf, Germany; s.antonov@mpie.de \\ * Correspondence: yjsu@ustb.edu.cn
}

Received: 15 February 2020; Accepted: 2 March 2020; Published: 6 March 2020

\begin{abstract}
The low yield strength ( 300 MPa) of twinning-induced plasticity (TWIP) steels greatly limits their structural applications in the industrial field. Conventional strengthening mechanisms usually cause an enhancement of yield strength but also a severe loss of ductility. In this research, gradient substructures were introduced in the Fe-22Mn-0.6C TWIP steels by different pre-torsional deformation in order to overcome the above limitations. The substructure evolution, mechanical properties, and their origins in gradient-substructured (GS) TWIP steels were measured and compared by electron backscattered diffraction (EBSD), monotonous and loading-unloading-reloading (LUR) tensile tests. It was found that a simple torsional treatment could prepare gradient twins and dislocations in coarse-grained TWIP steel samples depending on torsional strain. The uniaxial tensile tests indicated that a superior combination of high yield strength, high ultimate strength, and considerable ductility was simultaneously obtained in the GS samples. The high yield strength and high ultimate tensile strength were attributed to synergetic strengthening mechanisms, viz., dislocation strengthening, due to the accumulation of high density of dislocations, and very high back stress strengthening due to gradient substructure distribution, which was accommodated through pile-ups of extra geometrically necessary dislocations (GNDs) across the sample-scale. Additionally, high ductility originated from gradient substructure-induced back stress hardening. The present study is also beneficial to the design efforts of high strength and high ductility of other heterogeneous-structured TWIP alloy systems.
\end{abstract}

Keywords: twinning-induced plasticity (TWIP) steels; strength; ductility; gradient substructures

\section{Introduction}

High manganese twinning-induced plasticity (TWIP) steels have attracted great attention from the automotive industry in recent years due to their outstanding combination of mechanical properties, viz., high ultimate tensile strength $(\sim 800 \mathrm{MPa})$, high ductility $(\sim 80 \%)$, and enhanced strain hardening capability [1,2]. However, one key factor limiting the structural applications of TWIP steels is their low yield strength, which is about $300 \mathrm{MPa}$, arising from their single-phase microstructure, where the sole strengthening mechanism is dislocation strengthening during the initial deformation stages [3]. The high yield strength is particularly important for avoiding overload conditions, while high ductility is important for absorbing the impact energy, which the automobile may be subjected to [1]. Up to now, tremendous research efforts have been made to improve the mechanical properties of TWIP steels 
by precipitation strengthening [4], solid-solution strengthening [5], dislocation strengthening [6], etc. These conventional strengthening mechanisms, based on the concept of a homogenous microstructure design, lead to either an insufficient enhancement of yield strength or a great loss of the material's ductility by sacrificing the strain hardenability.

Recent studies have shown that a heterogeneous microstructure design is an effective way to simultaneously strengthen and toughen materials and has been applied to various alloy systems, i.e., Ti alloys [7], brass [8], and Fe alloys [9,10]. The heterogeneous microstructure can be categorized into a multi-modal grain size microstructure, heterogeneous lamella structure, nano-twinned structure, gradient structure, etc. Among these categories, the gradient structure, i.e., gradient dislocation density, phase content, or grain size distribution, can be produced by a surface mechanical attrition treatment (SMAT) [11] and surface mechanical grinding treatment (SMGT) [12]. For TWIP steels, particularly in a Fe-10Mn-3Ni-0.6C TWIP thin steel sample, a good combination of high strength and ductility was obtained through the formation of a $50 \mu \mathrm{m}$ thick gradient substructure produced through the SMAT technique [11]. However, the limited depth of the gradient structure (about a few hundred microns) formed by the general processing ways hinders the applicability to large-scale components. Recently, pre-torsion deformation, which can introduce gradient microstructures at larger scales, has been used to obtain good mechanical properties of $\mathrm{Cu}$ [13], $\mathrm{Mg}$ [14], and steels [15]. Using the same method, Wei et al. [16] found that a synergy of strength and ductility can be achieved through gradient nanotwins in a dumbbell-shaped TWIP steel specimen. They explained this phenomenon using an activation of different twinning systems along the depth of the steel sample based on finite element (FE) simulation investigations. Zhao et al. [17] also studied the excellent mechanical properties of gradient nanograined materials using a strain gradient plasticity model. In their numerical model, they discussed the effects of grain size, geometrically necessary dislocations (GNDs), and back stress on tensile response. However, to the authors' knowledge, experimental characterizations of the substructure evolution, i.e., deformation twinning and GND densities, and a detailed quantification of their respective roles in strengthening and strain hardening of gradient-substructured (GS) TWIP steels during tensile deformation, are still rare. Therefore, this study aims to clarify the relationships among the sample processing, resulting microstructures, and mechanical properties of a GS TWIP steel in detail. We believe that the present study is also beneficial to the design efforts for high strength and high ductility in other heterogeneous-structured TWIP alloy systems.

\section{Materials and Methods}

\subsection{Material and Sample Preparation}

In the present study, an ingot of Fe-22Mn-0.6C TWIP steel with measured compositions of $21.56 \mathrm{wt} . \% \mathrm{Mn}, 0.56 \mathrm{wt} . \% \mathrm{C}$, and balanced Fe was prepared by vacuum induction melting. To alleviate Mn segregation, the ingot was homogenized at $1150{ }^{\circ} \mathrm{C}$ for $2 \mathrm{~h}$ in a protective argon gas atmosphere. Subsequently, hot-rolling was performed at temperatures between $1100{ }^{\circ} \mathrm{C}$ and $950^{\circ} \mathrm{C}$ to form a square plate with dimensions of $50 \mathrm{~mm} \times 50 \mathrm{~mm}$. Finally, the plate was solution annealed at $1050^{\circ} \mathrm{C}$ to ensure microstructural homogeneities.

Dumbbell-shaped samples with a gauge section of $4 \mathrm{~mm}$ in diameter and $28 \mathrm{~mm}$ in length were excised from the abovementioned plate. This sample condition was termed as coarse-grained. Subsequently, pre-torsion to $90^{\circ}, 180^{\circ}$, and $360^{\circ}$ was applied on the above coarse-grained samples using an MTS 809 axial/torsional testing machine to fabricate GS samples of different degrees. The details of the processing process can be found in Section 3.1.

\subsection{Mechanical Tests}

Quasi-static, monotonous, and loading-unloading-reloading (LUR) tensile tests were performed on the coarse-grained and GS samples at a strain rate of $1 \times 10^{-4} \mathrm{~s}^{-1}$ at room temperature using an MTS Landmark tensile testing machine. An extensometer was used to measure the strain during tensile 
testing. The resolutions of the stress and strain measurements were $1 \mathrm{MPa}$ and $1.0 \times 10^{-5}$, respectively. All tensile tests were repeated at least three times to ensure the reliability and reproducibility of the data.

\subsection{Substructural Characterizations}

\subsubsection{Electron Backscattered Diffraction (EBSD) Characterizations}

The microstructural analysis of the samples was performed using Zeiss Gemini SEM 500 equipped with a fully automatic Oxford Instruments Aztec 4.0 EBSD system (Oxford, UK) and a complementary metal-oxide semiconductor (CMOS)-based Symmetry EBSD detector (Oxford, UK). The following settings were used during EBSD data acquisition: $70^{\circ}$ sample tilt, $20 \mathrm{kV}$ accelerating voltage, $15 \mathrm{~mm}$ working distance, $60 \mu \mathrm{m}$ aperture size, $200 \mathrm{~nm}$ step size, and $1 \times 1$ binning, which provide extremely high spatial and angular resolutions under the measuring limit of the EBSD instrument. The present EBSD parameters provide a resolution of $\sim 0.05^{\circ}$ [18]. Prior to EBSD observations, the samples were mechanically polished followed by electro-polishing for approximately 30-40 s using an electrolyte of $85 \mathrm{vol} \%$ acetic acid and $15 \mathrm{vol} \%$ perchloric acid, and a voltage of $22 \mathrm{~V}$ at about $283 \mathrm{~K}$.

\subsubsection{Methodology for Obtaining Deformation Substructures}

Utilizing EBSD inverse pole figures (IPFs), the twin volume fraction, which is defined as the total area of the deformation twins per observed area, can be quantitatively measured as a function of the tensile deformation [19]. Similarly, the corresponding GND density can be further extracted by post-processing the EBSD-IPF maps. Based on Pantleon's approach [20], the EBSD measurements can provide the average crystallographic orientation within an individual pixel on a 2D surface map, such that the lattice curvature and Nye's dislocation density tensor can be derived from the orientation differences between adjacent points. Ignoring the elastic strain tensor, the Nye's tensor can be related to the curvature tensor by the following Equation:

$$
\alpha_{i k} \approx \delta_{j l k} g_{i j, l}
$$

where $\alpha_{i k}$ is the Nye's dislocation density tensor, $\delta_{j l k}$ is the permutation tensor, and $g_{i j, l}$ is the lattice curvature obtained from EBSD orientation data [21]. Nye's tensor is also represented by the Burgers vectors and the line vector for all dislocation types:

$$
\alpha_{i k}=\sum_{m=1}^{N} b_{i}^{(m)} l_{j}^{(m)} \rho^{(m)}
$$

where $\rho^{(m)}$ is the density of the individual type of dislocations, $b^{(m)}$ is the Burgers vector, and $l^{(m)}$ is the linear vector. There are 18 different dislocation types, including 12 edge dislocations and 6 screw dislocations, for FCC structures [21]. As lattice curvatures perpendicular to the surface cannot be measured in a 2D map, a unique solution cannot be obtained using the above equations. Thus, the L1 minimization procedure is used to obtain the GND density that fits the observed lattice curvatures well, based on minimizing the total dislocation energy. A detailed discussion about the GND density measuring method can be found in Refs. [22-25]. In the present paper, the GND calculation was performed on the basis of the aforementioned method via MATLAB.

\section{Results}

\subsection{Torsional Treatment}

In the present study, coarse-grained samples were pre-torsioned to prepare GS samples, as schematically shown in Figure 1a. This treatment was performed with torsion angles of $90^{\circ}$, $180^{\circ}$, and $360^{\circ}$ at a nominal torsion rate of $1^{\circ} / \mathrm{min}$. The representative torque versus torsion angle curves are shown in Figure $1 \mathrm{~b}$. For simplicity, the samples pre-torsioned to $90^{\circ}, 180^{\circ}$, and $360^{\circ}$, 
are labeled as PT90, PT180, and PT360, respectively. For torsional deformation, the shear strain in the sample can be simply calculated as follows [14]:

$$
\gamma=\frac{r \theta}{l_{0}}
$$

where $\gamma$ is shear strain, $r$ is the radial position from the center of the cross-section, $\theta$ is the torsion angle, and $l_{0}$ is the initial length of the cylinder. Following Equation (3), a strain gradient is generated, that is, the shear strain along the radial direction linearly increases from the center to the surface on the cross-section of the pre-torsional cylinder. As a result, a gradient substructure is formed along the radial position.

(a)

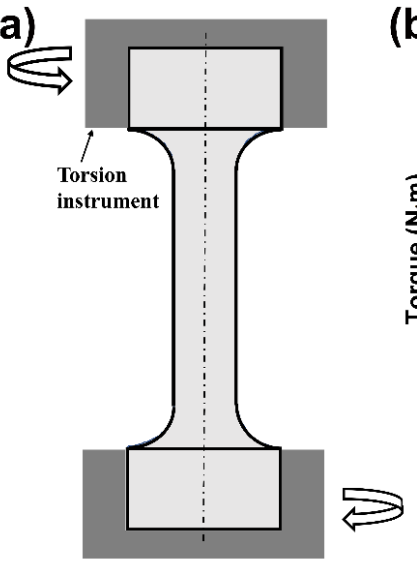

(b)

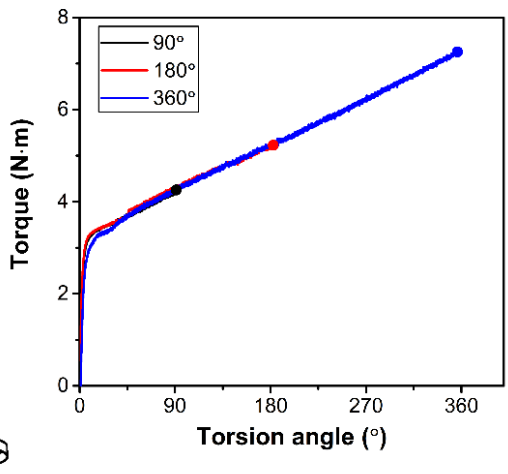

(c)

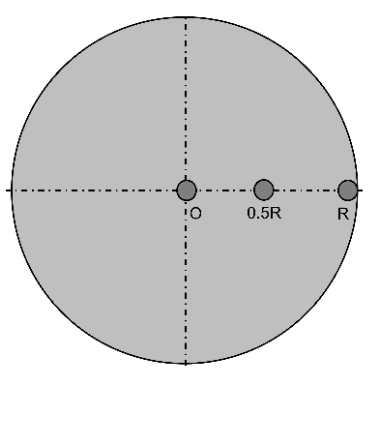

Figure 1. (a) Schematic diagram of pre-torsional deformation. (b) Measured torque versus torsion angle curve of Fe-22Mn-0.6C twinning-induced plasticity (TWIP) steel. (c) Sampling locations at the cross-section region.

\subsection{Gradient Substructures After Torsional Deformation}

To reveal the gradient substructures generated by torsional deformation, three positions along radial direction from the center to surface, viz., $r=0, r=0.5 R$, and $r=R$ (Figure $1 \mathrm{c}$ ), were characterized for the PT360 sample using EBSD. As exhibited in the IPF maps of Figure 2a-c, no deformation twins are generated in the center region $(r=0)$, primary twins are active at the position of $r=0.5 R$, and two twin systems simultaneously appear in the outermost layer $(r=R)$ due to an occurrence of the largest shear plastic strain. Figure $2 \mathrm{~d}$ shows that there are no deformation twins in the coarse-grained sample. The average twin volume fraction was quantitatively obtained using the EBSD-IPF maps, as described in Figure 2e. To ensure the statistical reliability of the results, multiple maps at each position were collected to obtain the abovementioned information. The results indicate that the twin volume fraction gradually increases from $0 \%$ to $12.5 \%$ along the radial direction of the PT360 sample.

Figure $3 a-c$ shows the corresponding GND density distribution maps for the PT360 sample. The transition from blue to red color in these maps represents a change of the GND density from 13.5 to 15.5 in $\log _{10}$ scale of lines per square meter. The amount of bright (red and yellow) regions increases from the center to the surface of the cross-section, indicating a gradual increase of the GND density along the radial direction across the sample-scale. In addition, the color distribution is non-homogeneous in each map, which reflects that the GND densities are non-homogeneously distributed within the grains, and are primarily concentrated at/near grain boundaries and twin boundaries. Figure $3 \mathrm{~d}$ presents the corresponding GND density map in the processed coarse-grained sample. Figure 3e shows the average value $\left(\rho_{\text {ave }}\right)$ of GND density, which is $0.5 \times 10^{14} \mathrm{~m}^{-2}, 1.3 \times 10^{14} \mathrm{~m}^{-2}$, and $3.0 \times 10^{14} \mathrm{~m}^{-2}$ at a position of $r=0,0.5 R$, and $R$ for the PT360 sample, respectively, and $0.4 \times 10^{14} \mathrm{~m}^{-2}$ for the coarse-grained sample. A much higher GND density appears in the near-surface regions $(r=R)$ due to an occurrence of the largest shear plastic strain and the formation of numerous deformation twin 
boundaries, which hinder the dislocation motion and locally induce an additional accumulation of GNDs. Therefore, the GS TWIP steels have a gradual increase in twin volume fraction and dislocation density along the radial direction.
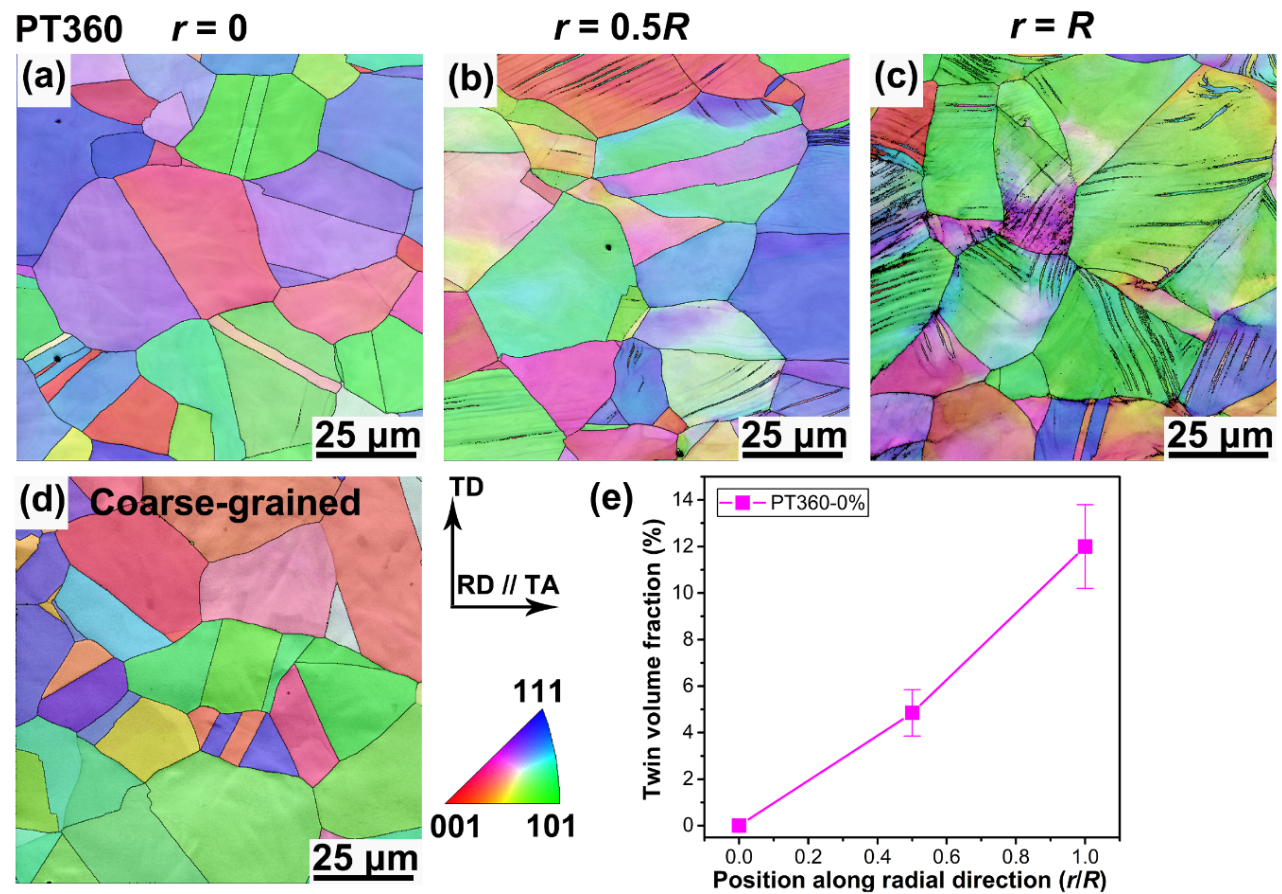

Figure 2. Characterization of the gradient twin substructure. Representative electron backscattered diffraction (EBSD) - inverse pole figure (IPF) maps at a position of (a) $r=0,(\mathbf{b}) r=0.5 R$, and (c) $r=$ $R$ on the cross-section of the PT360 sample. (d) Representative EBSD_IPF map for a coarse-grained sample. (e) Evolution of the twin volume fraction as a function of position.
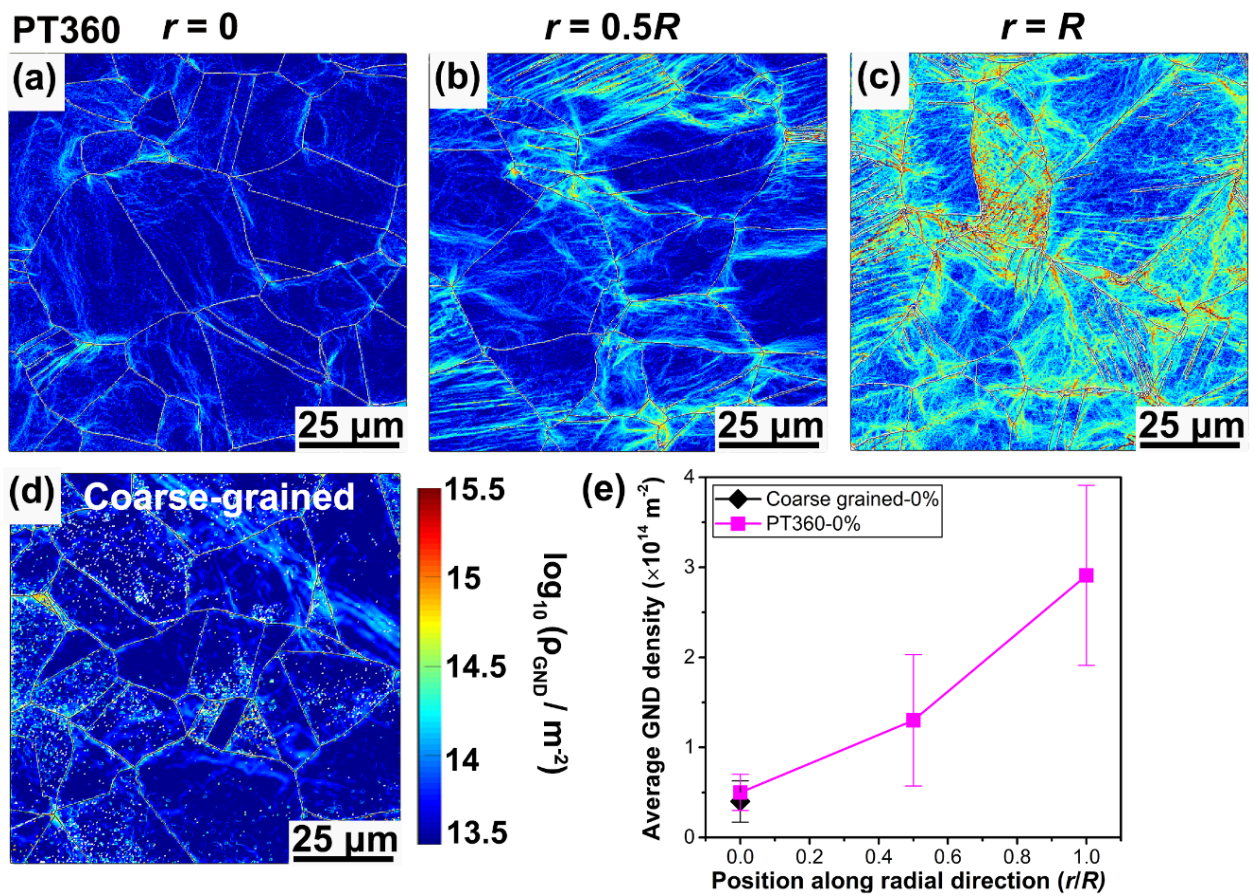

Figure 3. Representative EBSD-measured geometrically necessary dislocation (GND) distribution maps on the cross-section of the PT360 sample at a position of (a) $r=0,(\mathbf{b}) r=0.5 R$, and (c) $r=R$. (d) Representative GND distribution map for a coarse-grained sample. (e) Evolution of averaged GND density as a function of position. 


\subsection{Tensile Properties}

Figure 4a presents the tensile engineering stress-strain curves of coarse-grained and GS (PT90, PT180, and PT360) samples. Some specific tensile properties as a function of torsion angle are summarized in Figure 4b. Obviously, pre-torsional processing can significantly improve the yield strength $\left(\sigma_{y s}\right)$ and ultimate tensile strength $\left(\sigma_{u t s}\right)$ of the present Fe-22Mn-0.6C TWIP steel, while retaining the remarkable ductility. The $\sigma_{y s}$ increases from $321 \mathrm{MPa}$ for the coarse-grained sample to 413, 487, and $577 \mathrm{MPa}$ for the PT90, PT180, and PT360 samples, respectively. Meanwhile, the $\sigma_{u t s}$ increases from $949 \mathrm{MPa}$ for the coarse-grained sample to 974, 1012, and $1093 \mathrm{MPa}$ for the PT90, PT180, and PT360 samples, respectively. Particularly, the PT360 sample still retains a high uniform elongation $\left(\varepsilon_{u e}\right)$, viz., $60 \%$. The tensile properties achieved in this research are also compared with other TWIP steels reported in Refs. [4-6,10,26,27], as indicated in Figure 4c,d. The comparisons among them indicate a superior combination of yield strength, ultimate tensile strength, and tensile ductility in the present TWIP steels with gradient twin and dislocation substructures. For example, Kang et al. [4] prepared a precipitation-containing Fe-18Mn-0.6C-1.5 Al TWIP steel, but the $\varepsilon_{u e}(45 \%), \sigma_{y s}(500 \mathrm{MPa})$, and $\sigma_{u t s}(900 \mathrm{MPa})$ are about $25 \%, 13 \%$, and $18 \%$ lower than those of the PT360 sample, respectively. Kalsar et al. [11] produced a cold rolled Fe-10Mn-0.5C-3Ni TWIP steel with $\sigma_{y s}$ of $550 \mathrm{MPa}$, which is comparable to that of the PT360, but its $\varepsilon_{u e}$ is $10 \%$, which is $83 \%$ lower than that of the PT360 sample. As a result, it is reasonable to note that the GS design can be reasonably used to enhance strength with a moderate loss of ductility, providing a synergy between strength and ductility.
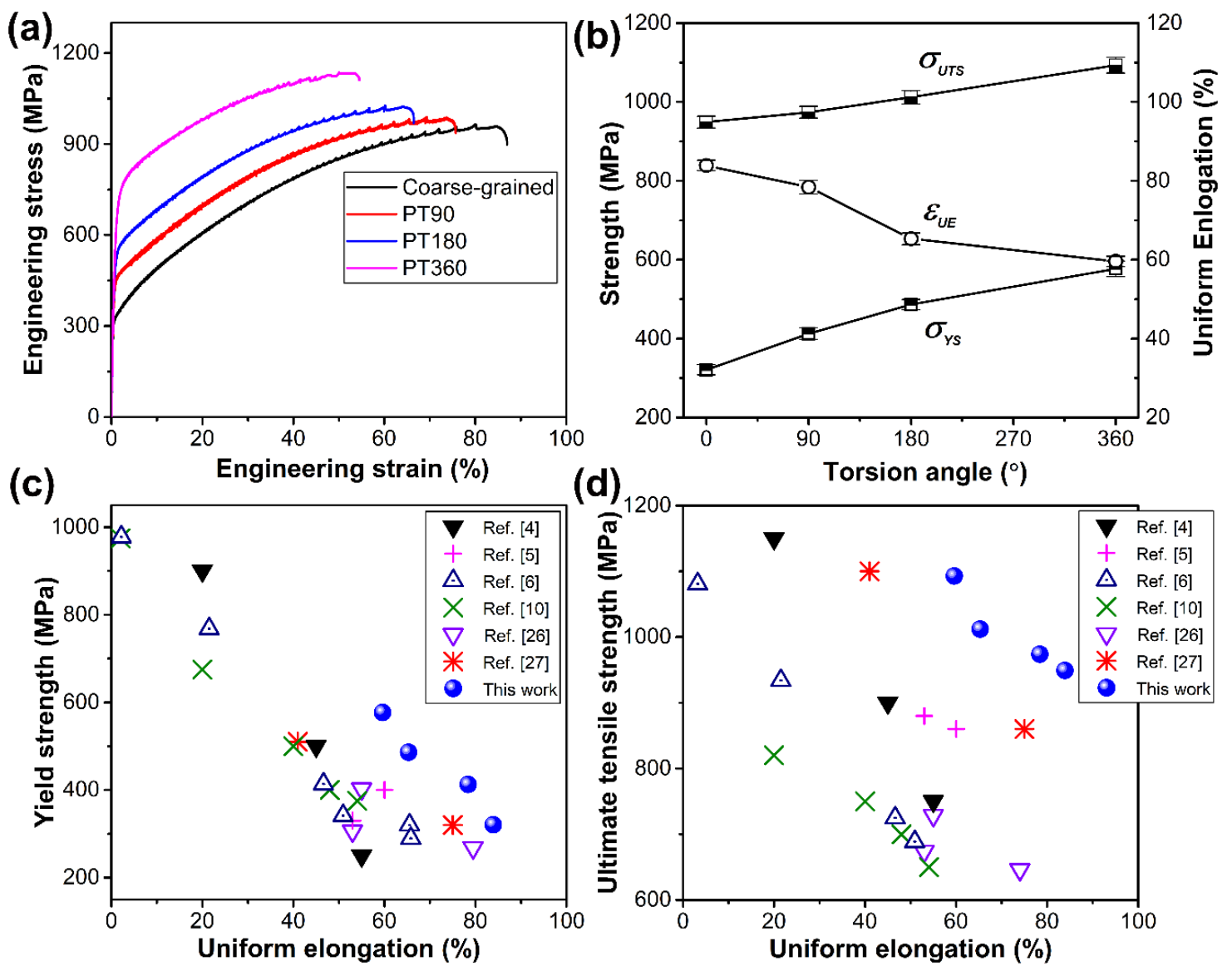

Figure 4. Mechanical properties of coarse grained and gradient-substructured (GS) (PT90, PT180, and PT360) samples. (a) Engineering stress-strain curves. (b) Yield strength, ultimate tensile strength, and uniform elongation as a function of the torsion angle. (c) Yield strength versus uniform elongation. (d) Ultimate tensile strength versus uniform elongation of pre-torsional samples compared to other TWIP steel samples reported in the references. 


\subsection{Substructural Evolution During Tension}

In order to understand the relations between mechanical properties and underlying deformation mechanism of the GS TWIP steel samples, the cross-sectional microstructures along the radial direction $(r=0,0.5 R, R)$ of the coarse-grained and PT360 samples after tensile deformation were characterized and compared here. Figure 5 shows substructure distribution maps of the coarse-grained sample tensioned to an engineering strain of $20 \%$. It is clearly seen that twin and dislocation substructures were generated in the deformed coarse-grained sample. The grain boundaries and twin boundaries hinder the dislocation motion, leading to pile-ups of high GNDs. Compared with Figures $2 \mathrm{~d}$ and $3 \mathrm{~d}$, one can see that the twin volume fraction and the overall GND density increase obviously. Additionally, there are no obvious differences in substructures among different positions in the deformed coarse-grained sample. Figure 6 presents twin and GND density distribution maps along the radial direction of the 20\% tensioned PT360 sample. Similar to the prepared PT360 sample (Figures 2a-c and 3a-c), gradient substructures continue to exist in the tensioned PT360 sample, but much more deformation twins and GNDs appear after tensile deformation from a qualitative view.
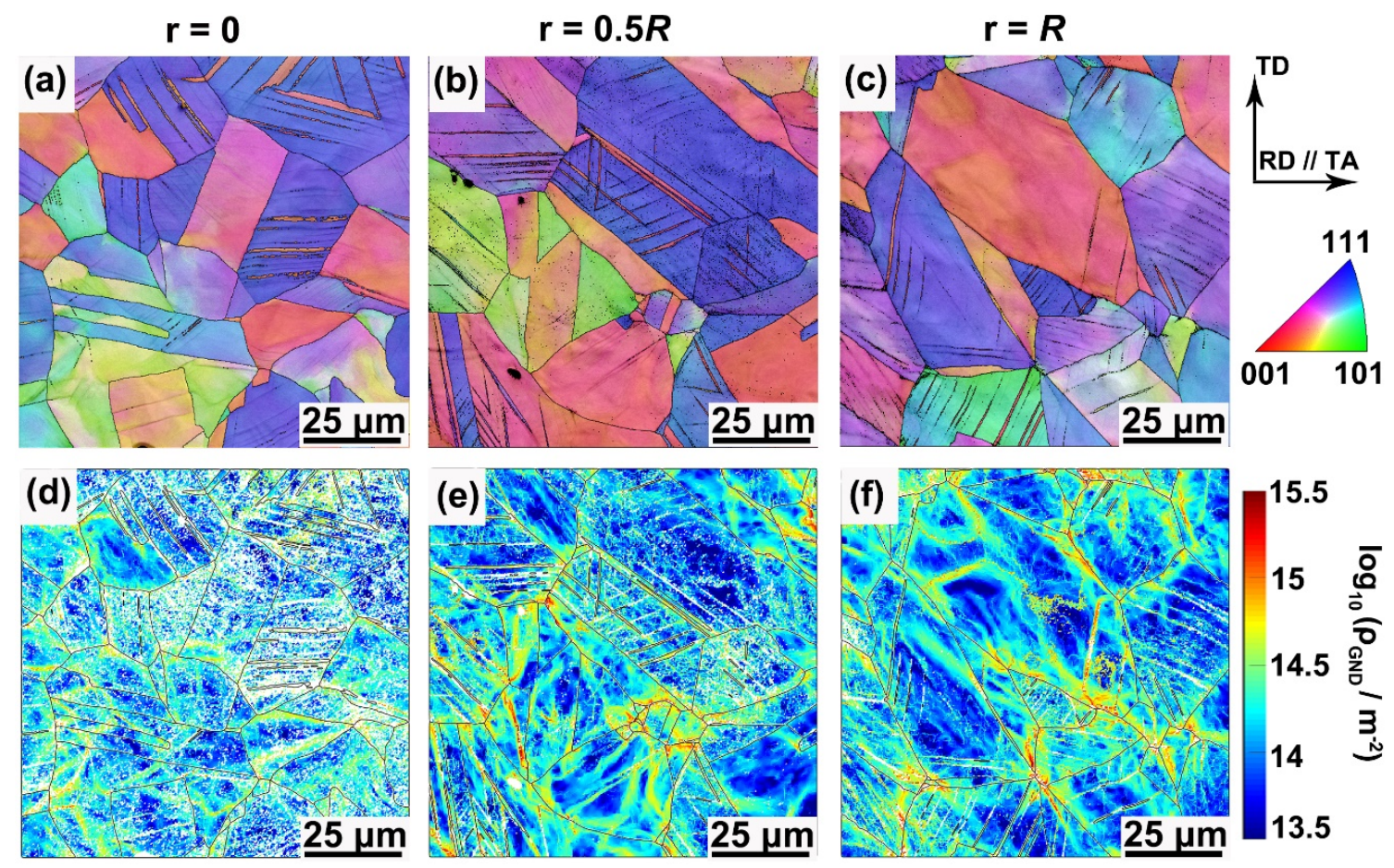

Figure 5. $(\mathbf{a}-\mathbf{c})$ Representative EBSD-IPF and (d-f) corresponding GND density distribution maps at an engineering strain of $20 \%$ for the coarse-grained sample.

The average twin volume fraction and GND density along the radial direction of two deformed samples were quantitatively extracted from the above maps (Figures 5 and 6). Figure 7a shows that at $20 \%$ engineering strain, the twin volume fraction at positions of $r=0,0.5 R$, and $R$ of the PT360 sample is approximately 2, 2.9, and 4.3 times that of the coarse-grained sample, respectively. Figure $7 \mathrm{~b}$ shows that the average GND density at positions of $r=0,0.5 R$, and $R$ of the PT360 sample is about 1.3, $1.5,2.5$ times that of the coarse-grained sample, respectively. Obviously, there is higher twin volume fraction and GND density in the bulk of the pre-tensioned PT360 TWIP steels. At positions of 0.5R and $R$, the higher substructure density in the PT360 sample can be attributed to a strain-hardened shell generated by the pre-torsional treatment. Within the center region $(r=0)$, the higher GND density and twin volume fraction of the PT360 sample during deformation are rationalized by two reasons. Due to variation in elastic constants and yield strength among grains, large amounts of deformation twins and GNDs are generated to achieve strain continuity across the grains in the coarse-grained 
and gradient samples [28]. In this case, these high GNDs are mainly blocked by grain boundaries and by newly generated twin boundaries during the tensile deformation (Figures 5 and 6). On the other side, in contrast to the coarse-grained samples, extra GNDs, and deformation twins are necessarily generated at the sample-scale to accommodate the large strain partitioning and strain gradients due to mechanical incompatibility between the hard shell and soft core of the PT360 sample during tensile deformation. These can explain why substructure densities in the center region are initially nearly the same in the undeformed coarse-grained and PT360 samples (Figure 2a,d and Figure 3a,d), but after tensile deformation, higher substructure densities appear in the center region of the PT360 sample compared to that of the coarse-grained sample (Figure 5a,d and Figure 6a,d).
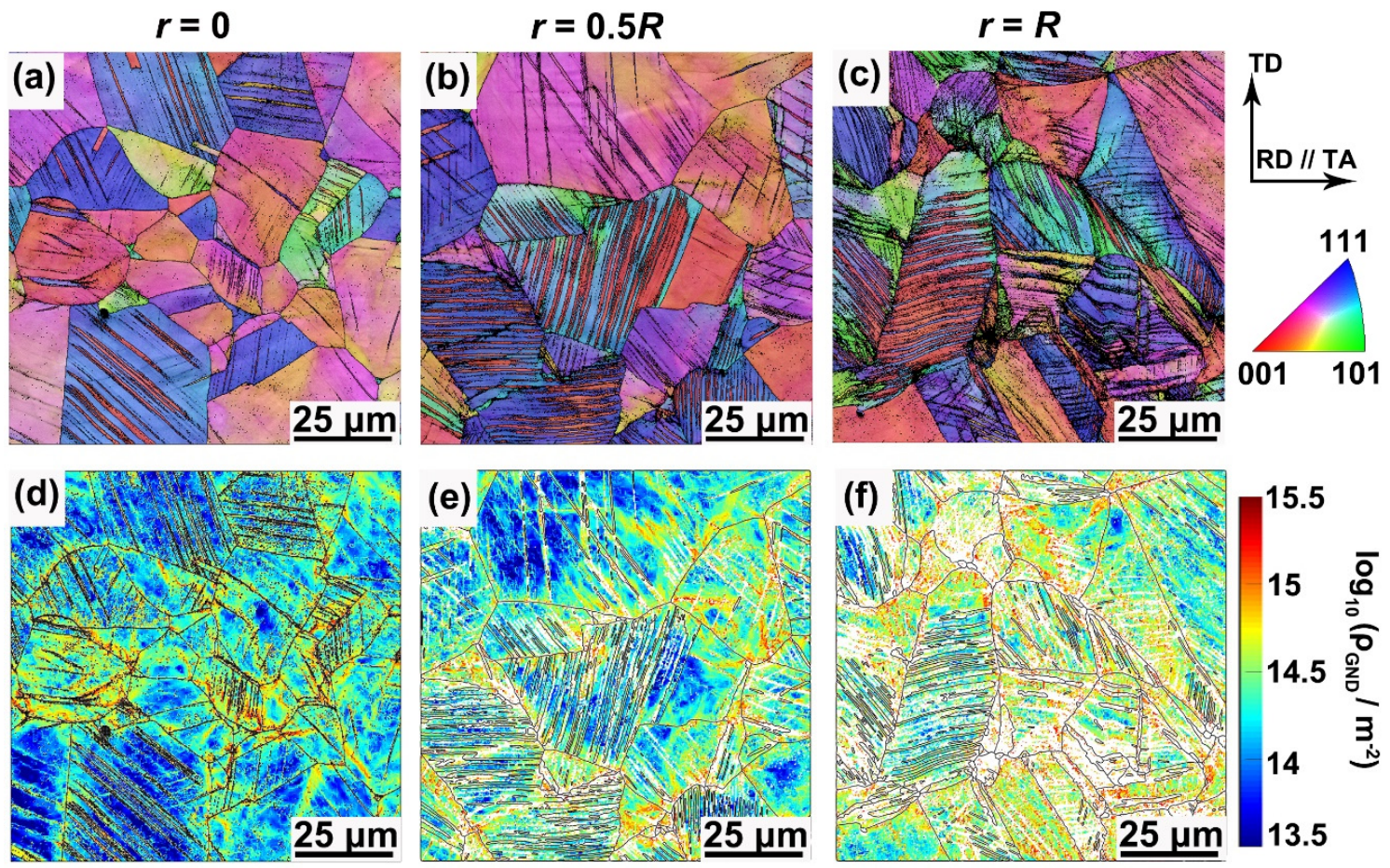

Figure 6. $(\mathbf{a}-\mathbf{c})$ Representative EBSD-IPF and (d-f) corresponding GND density distribution maps at an engineering strain of $20 \%$ for the PT360 sample.
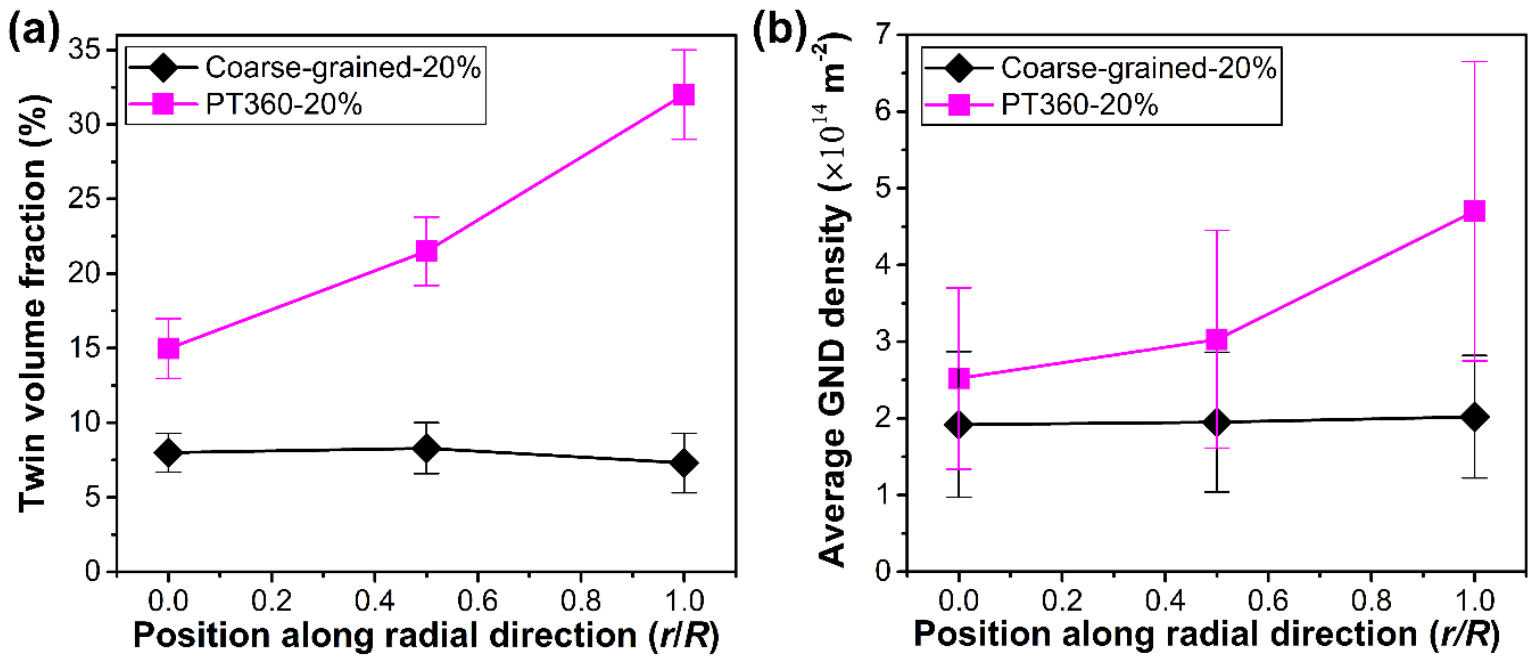

Figure 7. Evolution of (a) average twin volume fractions and (b) averaged GND densities as a function of positions for coarse-grained and PT360 samples pre-strained to $20 \%$ engineering strain. 


\section{Discussion}

\subsection{Dislocation Strengthening}

According to Ashby's theory, the total dislocation density is the sum of the GND density and statistically stored dislocation (SSD) density [29]. The flow stress due to dislocation strengthening $\left(\sigma_{\text {dislocation }}\right)$ can be described by Taylor's Equation as follows [30]:

$$
\sigma_{\text {dislocation }}=M a G b \sqrt{\left(\rho_{G}+\rho_{S}\right)},
$$

where $M$ is the Taylor factor, $G$ is the shear elastic modulus, $b$ is the magnitude of the Burger's vector, $\alpha$ is the short-range interaction coefficient between dislocations, $\rho_{G}$ is the GND density, and $\rho_{S}$ is the SSD density. Based on some geometrical assumptions, Ashby believed that the GND density increased with reducing the dislocation mean free path (MFP) and increasing the shear strain in materials, while the SSD density was nearly proportional to the shear strain squared [29]. In the present TWIP steel, the dislocation MFP depends on grain size and twin volume fraction. Thus, more GNDs (Figure 3e) can be generated near the surface region of the pre-torsioned GS TWIP steel due to combined effects of larger torsional strain (Equation (3)) and more deformation twins (Figure 2e). After 20\% tensile strain, there are much more deformation twins and GNDs in the GS TWIP steel than in the coarse-grained TWIP steel (Figure 7). In addition to the extra GNDs, a higher density of SSD should also form in the GS TWIP steel [17,29]. Following Equation (4), higher dislocation strengthening causes higher yield strength and tensile strength.

\subsection{Back Stress Induced Strengthening and Hardening}

GNDs not only contribute to dislocation strengthening as individual obstacles, but also generate a long-range back stress, which impedes dislocation emission from the dislocation sources [31,32]. In other words, higher flow stress is needed to overcome this field to sustain further plastic deformation. Several studies have reported that high back stress induces strengthening and hardening in heterogeneous-structured materials [8,33-36]. To measure the back stress generated during tensile deformation, LUR tests were performed on the GS (PT90, PT180, PT360) and coarse-grained samples. Each specimen was repeatedly loaded and unloaded at engineering strain levels of 2\%, 5\%, 10\%, 15\%, $20 \%, 30 \%$, and $40 \%$ (corresponding true strains of $0.02,0.05,0.095,0.14,0.18,0.26,0.33$ ), as exhibited in LUR tensile testing curves (Figure 8a). Figure 8 b shows a typical LUR loop at 0.14 true strain for each specimen. The hysteresis loop is evidence of the occurrence of a reverse yield when the overall applied stress is still in tension during unloading. As schematically depicted in Figure $8 \mathrm{c}$, the back stress $\left(\sigma_{b}\right)$ can be calculated following the method proposed by Yang et al. [37]:

$$
\sigma_{b}=\frac{\sigma_{r}+\sigma_{u}}{2}
$$

where $\sigma_{r}$ and $\sigma_{u}$ is the reloading yield stress and unloading yield stress, respectively. The $\sigma_{r}$ and $\sigma_{u}$ in each hysteresis loop can be determined by the point at which the effective elastic modulus $\left(E_{\text {eff }}\right)$ is reduced by $10 \%$, which was also adopted in some recent studies [8,34]. Figure $8 \mathrm{~d}$ exhibits the back stress measured using the above method with increasing true strain for the coarse-grained and GS (PT90, PT180, PT360) samples. The back stress increases as the strain increases for all samples, indicating that GNDs continuously accumulate during deformation. For comparison, the GS sample possesses much higher back stress than the coarse-grained sample at all studied strain ranges. The back stress induced hardening can be regarded as the slope of the curve in Figure 8d. Particularly, the PT360 sample possesses at least the double back stress of the coarse-grained sample prior to a true strain of 0.05 , and thereafter possesses nearly $60 \%$ higher back stress than the coarse-grained sample. Such a high back stress is in accord with pile-ups of more GNDs in the PT360 samples (Figure 7). As a result, the back stress strengthening and induced strain hardening further improved the flow stress and retained tensile ductility of the GS TWIP steel samples. 
(a)

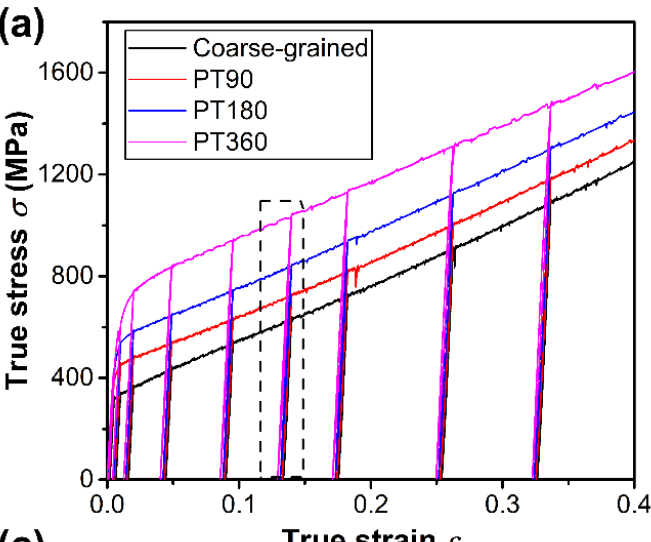

(c)

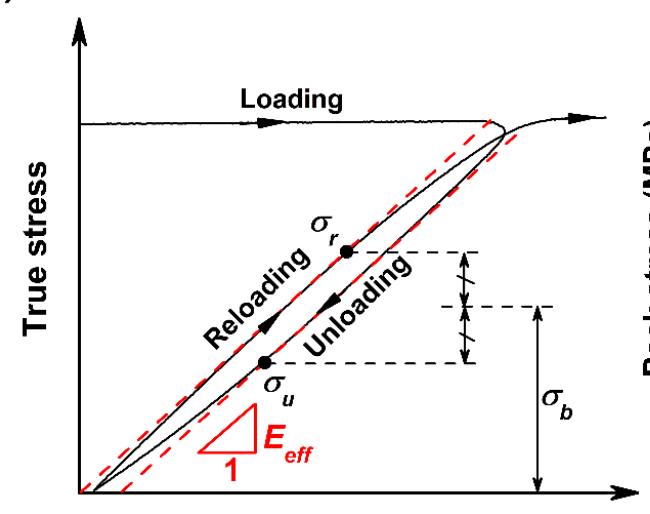

True strain

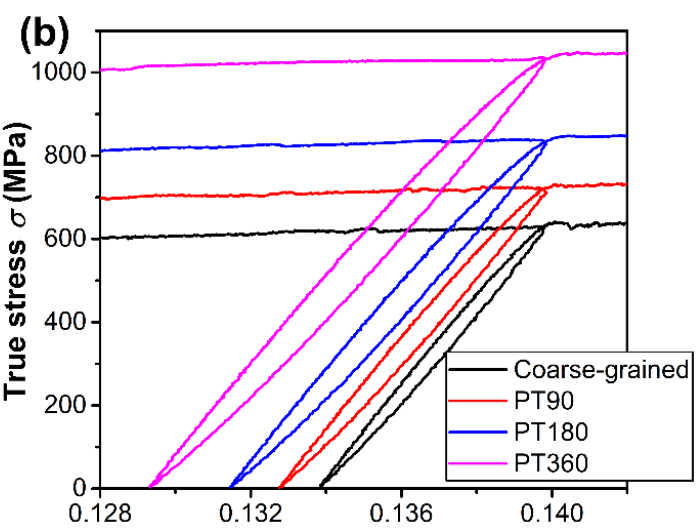

(d)

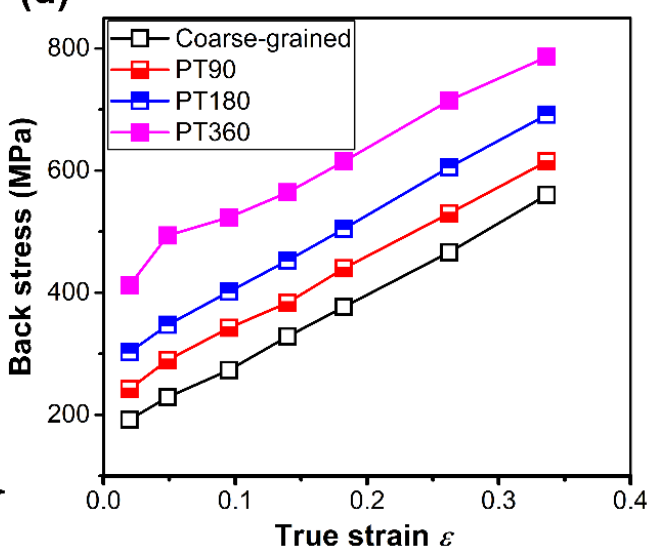

Figure 8. Back stress of coarse-grained and GS TWIP steel samples. (a) Loading-unloading-reloading (LUR) true stress-strain curves. (b) Enlarged view of typical hysteresis loops marked by a black dashed box in (a). (c) Schematic diagram illustrating the characteristics of hysteresis loops. (d) Variation of measured back stress with true strain.

\section{Conclusions}

In the present study, torsional treatment was used to generate gradient substructures with the aim of improving the yield strength and retaining the tensile ductility of TWIP steels. The main conclusions are drawn as follows:

(1) Torsional treatment induced gradient deformation twin and dislocation substructures in the TWIP steel. The twin and dislocation densities gradually increased along the radial direction from center to the surface of the GS TWIP steel sample;

(2) Tensile tests of the GS TWIP steel samples showed large improvements in the yield strength and ultimate tensile strength with only a modest compromise to the tensile ductility;

(3) Microstructural observations revealed that the gradient substructures still remained during tensile deformation, leading to a strain incompatibility and strain partitioning between the surface and center of the tensioned sample. As a result, more twins and GNDs were formed in the center region $(r=0)$ of the GS sample than in the bulk of the coarse-grained sample during tension;

(4) LUR tests showed that higher back stress was generated in the GS samples, which originated from the strain incompatibility between the surface and center across the sample-scale together with GND accumulation at/near grain and twin boundaries at the grain-scale;

(5) Consequently, the high yield and ultimate tensile strengths originated from a synergetic effect of dislocation strengthening and gradient substructure-induced back stress strengthening. Additionally, the high back stress induced high strain hardening, further leading to large tensile ductility of the GS TWIP steels. 
Author Contributions: Conceptualization, H.Z. and Y.S.; formal analysis, H.Z., C.Z., and Z.G.; funding acquisition, Y.S.; investigation, H.Z.; resources, H.Z., C.Z., and Z.G.; supervision, Y.S.; visualization, H.Z.; writing-original draft, H.Z., S.A., and Y.S.; writing-review \& editing, H.Z., S.A., and Y.S. All correspondence should be addressed to Y.S. All authors have read and agreed to the published version of the manuscript.

Funding: This research was funded by the National Key Research and Development Program of China (Grant No. 2016YFB0700505), the National Nature Science Foundation of China (Grant No. 51571028), and the 111 Project (Grant No. B170003).

Conflicts of Interest: The authors declare no conflict of interest.

\section{References}

1. Cooman, B.C.D.; Estrin, Y.; Kim, S.K. Twinning-induced plasticity (TWIP) steels. Acta Mater. 2018, 142, 283-362. [CrossRef]

2. Dobrzański, L.A.; Borek, W.; Mazurkiewicz, J. Mechanical Properties of High-Manganese Austenitic TWIP-Type Steel. Mater. Sci. Forum 2014, 783, 27-32. [CrossRef]

3. Jeong, K.; Jin, J.-E.; Jung, Y.-S.; Kang, S.; Lee, Y.-K. The effects of Si on the mechanical twinning and strain hardening of Fe-18Mn-0.6C twinning-induced plasticity steel. Acta Mater. 2013, 61, 3399-3410. [CrossRef]

4. Kang, S.; Jung, Y.-S.; Jun, J.-H.; Lee, Y.-K. Effects of recrystallization annealing temperature on carbide precipitation, microstructure, and mechanical properties in Fe-18Mn-0.6C-1.5Al TWIP steel. Mater. Sci. Eng. A 2010, 527, 745-751. [CrossRef]

5. Jin, J.-E.; Lee, Y.-K. Effects of Al on microstructure and tensile properties of C-bearing high Mn TWIP steel. Acta Mater. 2012, 60, 1680-1688. [CrossRef]

6. Santos, D.B.; Saleh, A.; Gazder, A.A.; Carman, A.; Duarte, D.M.; Ribeiro Érica, A.; Gonzalez, B.; Pereloma, E.V. Effect of annealing on the microstructure and mechanical properties of cold rolled Fe-24Mn-3Al-2Si-1Ni-0.06C TWIP steel. Mater. Sci. Eng. A 2011, 528, 3545-3555. [CrossRef]

7. Wu, X.; Yang, M.; Yuan, F.; Wu, G.; Wei, Y.; Huang, X.; Zhu, Y. Heterogeneous lamella structure unites ultrafine-grain strength with coarse-grain ductility. Proc. Natl. Acad. Sci. USA 2015, 112, 14501-14505. [CrossRef]

8. Fang, X.; He, G.; Zheng, C.; Ma, X.; Kaoumi, D.; Li, Y.; Zhu, Y. Effect of heterostructure and hetero-deformation induced hardening on the strength and ductility of brass. Acta Mater. 2020, 186, 644-655. [CrossRef]

9. Li, J.; Fang, C.; Liu, Y.; Huang, Z.; Wang, S.; Mao, Q.; Li, Y. Deformation mechanisms of 304L stainless steel with heterogeneous lamella structure. Mater. Sci. Eng. A 2019, 742, 409-413. [CrossRef]

10. Dini, G.; Najafizadeh, A.; Ueji, R.; Monir-Vaghefi, S. Improved tensile properties of partially recrystallized submicron grained TWIP steel. Mater. Lett. 2010, 64, 15-18. [CrossRef]

11. Kalsar, R.; Suwas, S. A novel way to enhance the strength of twinning induced plasticity (TWIP) steels. Scr. Mater. 2018, 154, 207-211. [CrossRef]

12. Chen, L.; Cao, T.; Wei, R.; Tang, K.; Xin, C.; Jiang, F.; Sun, J. Gradient structure design to strengthen carbon interstitial Fe40Mn40Co10Cr10 high entropy alloys. Mater. Sci. Eng. A 2020, 772, 138661. [CrossRef]

13. Guo, N.; Song, B.; Yu, H.; Xin, R.; Wang, B.; Liu, T. Enhancing tensile strength of Cu by introducing gradient microstructures via a simple torsion deformation. Mater. Des. 2016, 90, 545-550. [CrossRef]

14. Song, B.; Pan, H.; Chai, L.J.; Guo, N.; Zhao, H.; Xin, R. Evolution of gradient microstructure in an extruded AZ31 rod during torsion and annealing and its effects on mechanical properties. Mater. Sci. Eng. A 2017, 689, 78-88. [CrossRef]

15. Guo, N.; Zhang, Z.; Dong, Q.; Yu, H.; Song, B.; Chai, L.J.; Liu, C.; Yao, Z.; Daymond, M.R. Strengthening and toughening austenitic steel by introducing gradient martensite via cyclic forward/reverse torsion. Mater. Des. 2018, 143, 150-159. [CrossRef]

16. Wei, Y.; Li, Y.; Zhu, L.; Liu, Y.; Lei, X.; Wang, G.; Wu, Y.; Mi, Z.; Liu, J.; Wang, H.; et al. Evading the strength-ductility trade-off dilemma in steel through gradient hierarchical nanotwins. Nat. Commun. 2014, 5, 3580. [CrossRef]

17. Zhao, J.; Lu, X.; Yuan, F.; Kan, Q.; Qu, S.; Kang, G.; Zhang, X. Multiple mechanism based constitutive modeling of gradient nanograined material. Int. J. Plast. 2020, 125, 314-330. [CrossRef]

18. Goulden, J.; Trimby, P.; Bewick, A. The Benefits and Applications of a CMOS-based EBSD Detector. Microsc. Microanal. 2018, 24, 1128-1129. [CrossRef] 
19. Renard, K.; Jacques, P. On the relationship between work hardening and twinning rate in TWIP steels. Mater. Sci. Eng. A 2012, 542, 8-14. [CrossRef]

20. Pantleon, W. Resolving the geometrically necessary dislocation content by conventional electron backscattering diffraction. Scr. Mater. 2008, 58, 994-997. [CrossRef]

21. Kundu, A.; Field, D. Geometrically Necessary Dislocation Density Evolution in Interstitial Free Steel at Small Plastic Strains. Met. Mater. Trans. A 2018, 49, 3274-3282. [CrossRef]

22. Muránsky, O.; Balogh, L.; Tran, M.; Hamelin, C.; Park, J.-S.; Daymond, M. On the measurement of dislocations and dislocation substructures using EBSD and HRSD techniques. Acta Mater. 2019, 175, $297-313$.

23. Han, Q.; Rui, S.-S.; Qiu, W.; Ma, X.; Su, Y.; Cui, H.; Zhang, H.; Shi, H. Crystal orientation effect on fretting fatigue induced geometrically necessary dislocation distribution in Ni-based single-crystal superalloys. Acta Mater. 2019, 179, 129-141. [CrossRef]

24. Zhu, C.; Harrington, T.; Gray, G.T.; Vecchio, K.S. Dislocation-type evolution in quasi-statically compressed polycrystalline nickel. Acta Mater. 2018, 155, 104-116. [CrossRef]

25. Jiang, J.; Britton, T.B.; Wilkinson, A.J. Evolution of dislocation density distributions in copper during tensile deformation. Acta Mater. 2013, 61, 7227-7239. [CrossRef]

26. Wang, K.; Wei, A.; Shi, Z.; Chen, X.; Lin, J.; Tong, X.; Tao, Z.; Chen, X. The preparation and performance of grain size gradient TWIP steel fabricated by laser heat treatment. Mater. Sci. Eng. A 2019, 743, 294-300. [CrossRef]

27. Xiong, T.; Zheng, S.; Zhou, Y.; Pang, J.; Jin, Q.; Ge, H.; Zheng, X.; Yang, L.; Beyerlein, I.; Ma, X. Enhancing strength and thermal stability of TWIP steels with a heterogeneous structure. Mater. Sci. Eng. A 2018, 720, 231-237. [CrossRef]

28. Gil Sevillano, J. Geometrically necessary twins and their associated size effects. Scr. Mater. 2008, 59, $135-138$. [CrossRef]

29. Ashby, M.F. The deformation of plastically non-homogeneous materials. Philos. Mag. 1970, 21, $399-424$. [CrossRef]

30. Hansen, N. Polycrystalline strengthening. Met. Mater. Trans. A 1985, 16, 2167-2190. [CrossRef]

31. Hansen, L.T.; Fullwood, D.T.; Homer, E.R.; Wagoner, R.H.; Lim, H.; Carroll, J.D.; Zhou, G.; Bong, H.J. An investigation of geometrically necessary dislocations and back stress in large grained tantalum via EBSD and CPFEM. Mater. Sci. Eng. A 2020, 772, 138704. [CrossRef]

32. Lin, P.; Nie, J.; Liu, Z.; Zhuang, Z. Study of two hardening mechanism caused by geometrically necessary dislocations in thin films with passivation layer. Int. J. Solids Struct. 2019, 160, 59-67. [CrossRef]

33. Shin, S.; Zhu, C.; Zhang, C.; Vecchio, K.S. Extraordinary strength-ductility synergy in a heterogeneous-structured $\beta$-Ti alloy through microstructural optimization. Mater. Res. Lett. 2019, 7, 467-473. [CrossRef]

34. Lee, H.H.; Yoon, J.I.; Park, H.K.; Kim, H.S. Unique microstructure and simultaneous enhancements of strength and ductility in gradient-microstructured $\mathrm{Cu}$ sheet produced by single-roll angular-rolling. Acta Mater. 2019, 166, 638-649. [CrossRef]

35. Wang, Y.; Huang, C.; Fang, X.; Höppel, H.W.; Göken, M.; Zhu, Y. Hetero-deformation induced (HDI) hardening does not increase linearly with strain gradient. Scr. Mater. 2020, 174, 19-23. [CrossRef]

36. Huang, C.; Wang, Y.; Ma, X.; Yin, S.; Höppel, H.W.; Goken, M.; Wu, X.; Gao, H.; Zhu, Y. Interface affected zone for optimal strength and ductility in heterogeneous laminate. Mater. Today 2018, 21, 713-719. [CrossRef]

37. Yang, M.; Pan, Y.; Yuan, F.; Zhu, Y.; Wu, X. Back stress strengthening and strain hardening in gradient structure. Mater. Res. Lett. 2016, 4, 145-151. [CrossRef]

(C) 2020 by the authors. Licensee MDPI, Basel, Switzerland. This article is an open access article distributed under the terms and conditions of the Creative Commons Attribution (CC BY) license (http://creativecommons.org/licenses/by/4.0/). 